\title{
Improving heart rate recovery/deceleration as a modality for public health and athletic performance
}

\begin{abstract}
Objectives: The purpose of the present review study is to review the existing literature regarding the heart rate responses, specifically Heart Rate Deceleration (HRD) in both the public health and sports performance settings.

Search methods: A search was conducted on the limited body of research that exists in and around the world of public health and sports perforce specifically describing HRD. Literature gathered involved trails of comparative analysis with control groups in various exercise settings.

Main results :In an attempt to improve 'heart rate' in today's health and therapy disciplines, the greater focus is often on resting levels and modalities to improve one's resting heart rate. However, the activity of the heart post-exercise bout might be of greater interest for many health and sport practitioners. Specifically, heart rate recovery, or often described as heart rate deceleration has potent effects on mortality as well as athletic performance. Investigations have focused on the attention of vagal activity required to decelerate heart rate, and the relationship not only with prognostic sign for mortality risk, but also as a psychophysiological tool in sports performance settings.
\end{abstract}

Keywords: heart rate deceleration, heart rate activity, psychophysiological
Volume 2 Issue 2 - 2017

\author{
Christopher K Carroll \\ Department of Human Kinetics and Applied Health Science, \\ Bethel University, USA
}

Correspondence: Christopher K Carroll, Department of Human Kinetics and Applied Health Science, Bethel University, USA, Tel 65I5032459, Email carchrk@bethel.edu

Received: June 13, 2017 | Published: July 20, 2017
Abbreviations: HRD, heart rate deceleration LVEF, left ventricle ejection fraction

\section{Introduction}

Heart rate activity, specifically heart rate recovery and heart rate deceleration (HRD), is at the forefront of much public health and medical research. It is hypothesized that HRD post-exercise is linked to mortality and that a compromised HRD may be a powerful predictor of all-cause mortality. ${ }^{1}$ Historically, the focus of most of the medical research has investigated changes in heart rate activity during exercise and stress testing ${ }^{2}$ (Lauer et al., 1996; Sandvik et al., 1995). However, a heightened interest in the decline in heart rate after the discontinuation of exercise has recently drawn interest, particularly as it relates to public health in post infarction risk and mortality risks. ${ }^{3,4}$

\section{Methods}

The present study was undertaken an electronic search of practical and related journals, textbooks and reference lists along with a hands on search of published and unpublished works related to the subject in question. Both abstracts and full texts were evaluated.

\section{Discussion}

Increased heart rate during exercise is the result of decreased parasympathetic activity and increased sympathetic activation. ${ }^{5,6}$ The decline of heart rate after exercise is due to the reactivation of the parasympathetic nervous system. ${ }^{6,7}$ Specifically, HRD is a result of vagal reactivation. Due to the relationship between vagal activity and HRD, the relationship may be used as a decisive prognostic sign for mortality risk. ${ }^{1}$
Cole et al. ${ }^{1}$ analyzed HRD on 2428 patients and during the 6-year follow-up an atypical HRD was significantly predictive of death. Of the 213 deaths, over 50\% recorded abnormal HRD values. Interestingly, Cole et al. ${ }^{1}$ also discovered a strong correlation between HRD and exercise capacity in both men and women. Researchers noted that an athletic population showed improved HRD when compared to other populations.

Similar research links physical fitness, operationally defined by $\mathrm{VO}_{2 \max }$, with HRD. Goldsmith et al. ${ }^{8}$ and Goldsmith et al. ${ }^{9}$ show a relationship between vagal activation and modulation with maximal oxygen uptake in healthy males when age was controlled, the relationship between aerobic capacity and HRD is a vital key to bolstering not only public health interventions, but also has implications to sports performance.

Finally Bauer et al. ${ }^{3}$ and Guzizk et al. ${ }^{4}$ proposed heart rate variability analysis in separate modulations of acceleration and deceleration. These researchers postulated that the extent of the deceleration related heart rate variability is superior to the acceleration heart rate variability, which is widely established measure of left ventricle ejection fraction (LEVF). Moreover, the researchers indicated that deceleration capacity is a stronger, more pronounced predictor of mortality. Specifically, is better than LVEF, conventional measures of heart rate variability and the combination of both. Data collected by Bauer et al. ${ }^{3}$ and Guzizk et al. ${ }^{4}$ confirmed the deceleration capacity provides a measure of cardiac vagal modulations resulting in the cardio-protective role of vagal activity. 


\section{Heart rate recovery/deceleration and performance}

Heart rate deceleration (HRD) has been identified as a powerful psychophysiological tool in predicting sport performance. The psychophysiological ability to manage heart rate and heart rate activity has been connected to not only increase performance, but also to greater attention to a salient stimulus, increased reaction time and overall cognitive function and activity. ${ }^{10,11}$

Most competitive sports and in-game performance activities involve a preparatory period leading up to a critical juncture in a competition (i.e., a free throw in basketball, batting in baseball, snapping the ball in football). The preparatory period is thought to be a predictive marker of final performance. ${ }^{12,13}$ The ability to decrease heart rate to an optimal level during this period prior to the task at hand is assumed to be a vital component for optimal attention processes, and thus predictive of ideal performance. ${ }^{13,14}$ Most literature linking HRD and performance has investigated aiming proficiency, such as archery, ${ }^{13,15}$ rifle shooting, ${ }^{16}$ pistol shooting ${ }^{14}$ or golf putting. ${ }^{17,18}$ Greater HRD was revealed in elite level shooters and golfers when compared to less skilled archers and beginning golfers. ${ }^{17}$

Performance measures rely on postural balance and control to maintain reference while aiming. In a study by Cottyn et al., ${ }^{19}$ researchers investigated the effects of complex balance performance in reference to HRD. The authors found HRD to have a similar effect on the preparation phase of a balance beam acrobatic element in female gymnasts. However, results failed to show significant findings relating HRD and increased balance measures. ${ }^{19}$ Although this study did not show performance proficiency, it continued to support the previous literature related to neurocardiologic and physiologically mediated HRD. Nonetheless, performance research in sport has indicated HRD and performance are interrelated.

\section{Conclusion}

The connection between HRD and vagal modulation in public health settings and HRD and greater in-competition sport performance cannot go unnoticed. In a public health environment, empirical evidence exists directly linking HRD and mortality and aerobic capacity appears to be a significant factor in that connection..$^{3,4,8}$ Sport performance also appears to be enhanced with greater HRD as cited previously. However, the relationship between aerobic capacity and HRD has not been fully supported by empirical research for the anaerobic athlete and is an area of future investigation.

The data analyzed suggests that attention and concentration may be warranted to the improvement of HRD as a result of improved aerobic capacity. The overall increase of aerobic capacity/ $\mathrm{VO}_{2 \max }$ will not only improve performance outcome measures in athletic settings, but the simple increase of $\mathrm{VO}_{2 \max }$ in the public health world will improve vagal reactivation of the heart, thus the relationship may be used as a decisive prognostic sign for mortality risk. Practitioners can now assess and track aerobic capacity improvements and not solely rely on resting heart rate data as a predictor of health and mortality.

\section{Acknowledgements}

None.

\section{Conflict of interest}

The author declares no conflict of interest.

\section{References}

1. Cole CR, Blackstone EH, Pashkow FJ, Heart-rate recovery immediately after exercise as a predictor of mortality. $N$ Engl $J$ Med. 1999;341(18):1351-1357.

2. Ellestad MH, Wan MK. Predictive implications of stress testing: Follow-up of 2700 subjects after maximum treadmill stress testing. Circulation. 1975;51(2):363-369.

3. Bauer A, Kantelhardt JW, Barthel P, et al. Deceleration capacity of heart rate as a predictor of mortality after myocardial infarction: cohort study. Lancet. 2006;367:1671-1681.

4. Guzik P, Piskorski J, Barthel P, et al. Heart rate deceleration runs for post infarction risk prediction. Journal of Electrocardiology. 2012;45(1):70-76.

5. Arai Y, Saul JP, Albrecht P, et al. Modulation of cardiac autonomic activity during and immediately after exercise. Am J Physiol. 1989;256(1):H132-141.

6. Brooks GA, Fahey TD, Baldwin KM. Exercise physiology: Human bioenergetics and its applications 4th ed. USA: New McGraw Hill Education; 2005

7. Imai K, Sato H, Hori M, et al. Vagally mediated heart rate recovery after exercise is accelerated in athletes but blunted in patients with chronic heart failure. J Am Coll Cardiol. 1994;24(6):1529-1535.

8. Goldsmith RL, Bigger JT, Bloomfield DM, et al. Physical fitness as a determinant of vagal modulation. Med Sci Sports Exerc. 1997;29(6):812-817.

9. Goldsmith RL, Bloomfield DM, Rosenwinkel ET. Exercise and autonomic function. Coron Artery Dis. 2000;11(2):129-135.

10. Jennings JR, Wood CC. Cardiac cycle time effects on performance, phasic cardiac responses and their intercorrelation in choice reaction time. Psychophysiology. 1977;14(3):297-307.

11. Lacey BC, Lacey JI. Two-way communication between the heart and the brain: Significance of time within the cardiac cycle. American Psychologist. 1978;33(2):99-113.

12. Guillot A, Collet C, Dittmar A, et al. Psychophysiological study of the concentration period in shooting. Journal of Human Movement Studies. 2005;48(6):417-436.

13. Landers DM, Han M, Salazar W, et al. Effects of learning on electroencephalographic and electrocardiographic patterns in novice archers. International Journal of Sport Psychology. 1994;25(3):13-33.

14. Tremayne P, Barry RJ. Elite pistol shooters: Physiological patterning of best vs. worst shots. Int J Psychophysiol. 2001;41(1):19-29.

15. Robazza C, Bortoli L, Nougier V. Performance emotions in an elite archer: A case study. Journal of Sport Behaviour. 2000;23(2):144 163.

16. Konttinen N, Lyytinen H, Viitasalo J. Preparatory heart rate patterns in competitive rifle shooting. J Sports Sci. 1998;16(3):235-242.

17. Boutcher SH, Zinsser NW. Cardiac deceleration of elite and beginning golfers during putting. Journal of Sport \& Exercise Psychology. 1990;12(1):37-47. 
18. Hassmen P, Koivula N. Cardiac deceleration in elite golfers as modified by noise and anxiety during putting. Perceptual and Motor Skills. 2001;92(3s):947-957.
19. Cottyn J, Clercq D, Crombez G, et al. The role of preparatory heart rate deceleration on balance beam performance. $J$ Sport Exerc Psychol. 2008;30(2):159-170. 